\title{
Versatile mass excited impact oscillator
}

\author{
Marian Wiercigroch $(\mathbb{D}$ - Stephane Kovacs • \\ Shun Zhong • Dimitri Costa • Vahid Vaziri • \\ Marcin Kapitaniak • Ekaterina Pavlovskaia
}

Received: 24 April 2019 / Accepted: 12 November 2019 / Published online: 10 December 2019

(C) The Author(s) 2019

\begin{abstract}
This paper presents the design and the initial experimental results of a novel impact oscillator rig developed by the Centre of Applied Dynamic Research at the University of Aberdeen. In this rig, the excitation force is generated electromagnetically and it acts directly on the mass in contrast to the most of the experimental set-ups where the excitation passes through the structure. This significantly enhances flexibility of the system allowing to observe subtle phenomena. The evolution of the design from an initial concept to the finalised rig is discussed in details where a special attention is paid to the instrumentation and parameter identification which are important for the mathematical modelling. The initial experimental results demonstrate potentials of this rig to study fundamental impact phenomena, which have been observed in various engineering systems. They also indicate that this new rig can
\end{abstract}

M. Wiercigroch $(\varangle) \cdot$ S. Kovacs · S. Zhong · D. Costa ·

V. Vaziri · M. Kapitaniak · E. Pavlovskaia

Centre for Applied Dynamics Research, School of

Engineering, University of Aberdeen, King's College,

Aberdeen AB24 3UE, Scotland, UK

e-mail: m.wiercigroch@abdn.ac.uk

E. Pavlovskaia

e-mail: e.pavlovskaia@abdn.ac.uk

S. Zhong

School of Mechanical Engineering, Tianjin University,

Tianjin, China

D. Costa

Center for Nonlinear Mechanics, COPPE - Department of Mechanical Engineering, Universidade Federal do Rio de Janeiro, P.O. Box 68503, Rio de Janeiro, RJ 21941972, Brazil be a good platform for investigating nonlinear control methods.

Keywords Oscillator · Impacts · Vibration · Nonlinear dynamics $\cdot$ Experiments $\cdot$ Design

\section{Introduction}

Impacts appear in a wide range of real systems, and understanding of these nonlinear phenomena is essential for further improvement in machine design and drilling processes [1-4] amongst others. In some cases, impacts are imperative part of the system operation, but they also may result from ageing of parts, thermal deformation or design tolerances. For example, in rotor dynamics, effects of bearing clearances are very important, and they were investigated using Jeffcott rotor model [5] where the interactions between a mass imbalanced rotor and a snubber ring without preloading of the snubber ring [6] and with a preloading $[7,8]$ were considered, and different impacting scenarios depending on the regimes of operation of the rotor were described [9]. Another illustration of a practical relevance of impacts is the behaviour of mooring lines which are repeatedly slacken and then tighten again under wave forcing [10], and a small floating structure moored to a large one can unexpectedly modify the motion of the whole system. Impacting systems have been extensively studied theoretically both considering rigid [11-17] and soft constraints [18-20]. 
In previous experimental investigations, a common approach was to provide the system excitation through the base, as was done, for example, by Moon and Shaw in $[21,22]$. The considered system consisted of a cantilever beam clamped at one end and free at the other end, where the motion of the free end was constrained by an aluminium stop. The clamped end of the beam was attached to a large electromagnetic shaker and driven periodically. Bounded, non-periodic or chaotic vibration were observed experimentally and studied theoretically using impact oscillator model. The design of an experimental rig vertically excited by a shaker was presented by Sin and Wiercigroch in [23], and a comprehensive experimental study of this system is conducted by the same authors in [24]. The rig comprises of a steel mass supported by a pair of parallel leaf springs to maintain the pure vertical motion of the mass. It impacts upon the screws located at the top of the secondary beams positioned on one or both sides of the oscillating mass. This rig developed in the Centre for Applied Dynamics Research at the University of Aberdeen has been used for numerous studies including ones by Ing et al. in [25-27], where global system bifurcations and the narrow band of chaos appearing close to grazing orbits were investigated. Another example of a base driven system is provided by an impacting pendulum with horizontal excitation presented by George et al. [28]. This system was composed of a hard rubber ball attached to a rigid-arm pendulum coming in contact with a fixed barrier, and the angular position of the pendulum was recorded. The transition behaviour of the system before settling was the focus of this investigations, and co-existing attractors and super persistent chaotic transients were studied.

Recently, several studies were focused on multistability of nonlinear dynamical systems. Multistability is observed when depending on the initial conditions, the system response can converge towards one of a number of co-existing attractors. This interesting phenomenon occurs, for example, in rotor dynamics [29] or impact oscillators [30] with co-existing impacting and non-impacting regimes or in gear rattle systems [31]. Depending on the specific system requirements, switching between co-existing attractors either should be avoided to increase the operational lifetime or could be desired to rapidly bring the system from one stable state to another one while minimizing the control effort and transition time [30].
The latter can have useful applications in smart structures [32,33] or energy harvesters [34]. Several control methods have been applied on a double pendulum system in [35] and on a bi-stable truss in [36]. A review of these methods is presented in [37]. In order to implement, test and develop such control methods, there is a need for a new rig where control could be applied directly on the mass of the oscillator.

In this paper, the design of a new impact oscillator is presented, in which the excitation is applied directly on the mass. The mass of the oscillator is actuated by a magnetic field which causes mass to move in horizontal direction. Similar design with vertical magnetic levitation system used for energy harvesting has been developed by Kecik in [38], where the dynamics of a vertically excited magnet oscillating inside a coil is investigated. The coil was placed at mid-height of a plexiglass cylinder with two fixed magnets at its extremities. It was observed that when the amplitude of the magnet oscillations increases under external loading, the coupling between the magnetic field of the coil and the magnets actuating the mass results in a hardening behaviour of the system.

The rest of the paper is structured as follows. In Sect. 2, the design of a new mass excited impact oscillator is presented where configurations tested to improve the original system are discussed. The actuation process of the mass has been investigated, and several types of excitation methods have been compared to achieve an acceptable interplay between an excitation applied directly on the mass and the natural dynamical behaviour of the system. In the following section, identification of the system parameters is presented, and then, some preliminary experimental results are detailed and discussed in Sect. 4. Finally, concluding remarks are given.

\section{Rig design}

In this section, an evolution of the design of the new impact oscillator is outlined. First, an oscillator driven by a magnetic field is presented and the different components of the rig are discussed. Then, the successive modifications of the original design are described. 
Fig. 1 Initial design of the impact oscillator showing the following components: (1) base plate; (2) main support column; (3) square pillar; (4) leaf spring; (5) mass; (6) adjustable bracket; (7) secondary support impact beams; (8) bracket plates; (9) coils; and (10) base legs

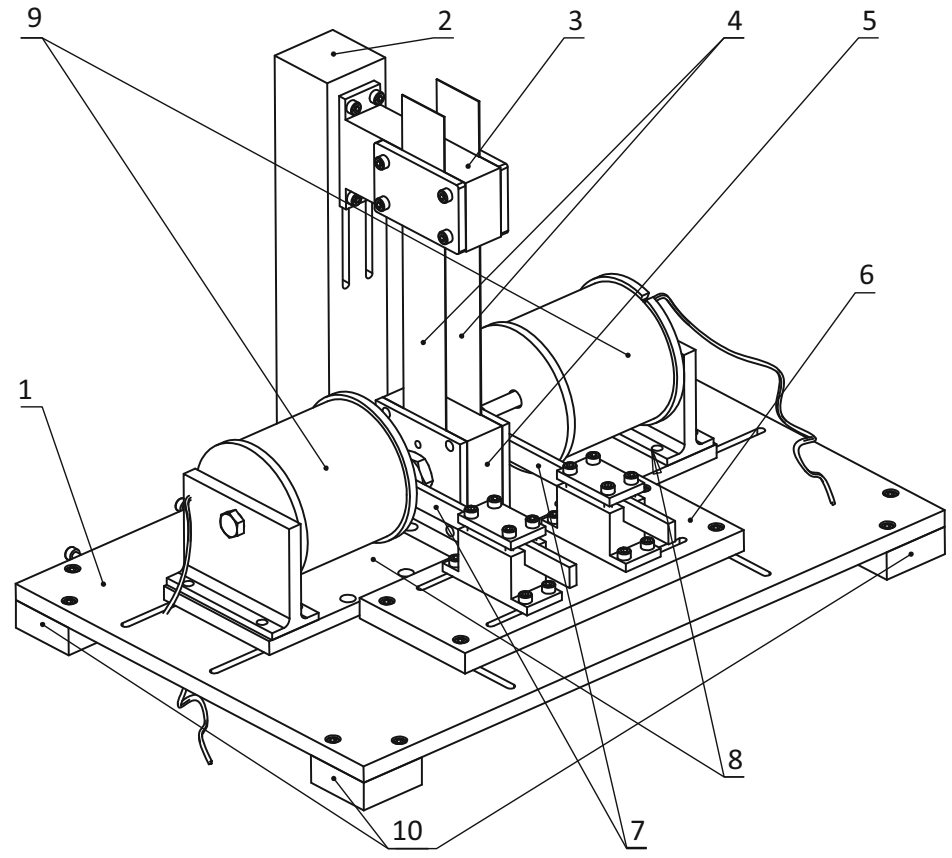

The square pillar, marked in Fig. 1 by number 3, can slide vertically along the main column so that the length of the leaf springs could be adjusted. This allows to vary considerably the value of stiffness, damping coefficients and natural frequency of the oscillator. The secondary support impact beams are also mounted on adjustable brackets (marked by number 6 ) so that the desired secondary stiffness could be achieved. The coils providing the excitation are attached to a slider (marked by number 8 ) which allows to move them closer or away from the mass depending on the requirements.

\subsection{Instrumentation of the rig}

There are three main sensors originally installed on the rig including an eddy current probe and two accelerometers, one for the motion of the oscillating mass and the other one for the support structure. An eddy current probe is positioned on top of the leaf springs. It records a voltage variation and allows to determine displacement of the mass by using an appropriately calibrated proportional coefficient. Such calibration procedure will be described in Sect. 3. The calibration is repeated when the length of the leaf spring is changed. The signal of displacement is then differentiated numerically to obtain the mass velocity. Two accelerometers, one attached to the mass and one placed at the top of the 
main column, record, respectively, the mass acceleration and the base acceleration. They are essential and complementary to measure the motion and residual signal such as the mechanical noise.

Once the components of the rig were manufactured and assembled together, the first set of experiments was carried out. They confirmed that the chosen set-up was not able to produce the required range of the excitation force amplitude and also that the supporting frame was not rigid enough to suppress structural vibration. Therefore, further modifications of the rig were undertaken and they are described in the following section.

\subsection{Improved design}

In this section, first the optimisation of the rig is discussed, and then, the improvement in the mechanical design is performed to reduce the noise in the structure resulting from impacts. In addition, various sensors have been added to the rig and a LabVIEW program was developed to drive the system and to control the magnetic force of excitation. The LabVIEW data acquisition system allows to control the input signal according to the force threshold and captures the data automatically once the user defined the parameters of the experiments.

\subsubsection{Excitation force}

Several different types of excitation methods have been compared in the current study. First, the magnetic field was strengthen by increasing the diameter of the iron cores and by adding two cylindrical magnets, attached to the mass from both sides. This modification aimed to generate a constant magnetic field between the coils and the mass. The signals recorded were clear and had reasonable amplitude but were not sinusoidal because of uncertain interactions between the first and the second coils. To obtain a harmonic signal, the use of one coil only became necessary, but this resulted in a reduced range of the excitation amplitude.

A constant and uniform magnetic field was also desired, and this was achieved by positioning coil poles close enough to the mass. The diameter of the coil was chosen to be ten times larger than the distance to the mass of the oscillator, as in this case the magnetic field applied on the mass could be considered direct and constant. Figure 2 a shows a coil of $160 \mathrm{~mm}$ diameter filled with a huge steel core of $5 \mathrm{~kg}$ which was now selected for the experiments to generate a suitable excitation. However, to achieve the desired motion, the mass had to be smaller. Therefore, several different types of plastic masses have been designed and 3D printed. Figure $2 b$ presents an assembly used to obtain four different values of the oscillating mass. Different types of inserts were manufactured, and they are shown in Fig. $2 b$ (marked by numbers $2-5$ ). They were placed between the covers (marked by number 1 ), and the leaf springs were positioned between the covers and the inserts and were fixed by a number of screws. Steel mass (marked by number 2), 3D printed plastic perforated mass (number 3), 3D printed plastic mass with a cavity to add a square aluminium insert (number 4) and lightweight 3D printed plastic mass with two holes (number 5) were tested in the experiments.

A number of experiments were conducted using configurations with different masses, but for very light plastic mass the recorded signals contained significant noise which had to be heavily filtered. This resulted in a loss of data containing events such as impacts, and therefore, such a configuration was not acceptable. Different coil sizes and types of magnet have been tested to optimise the displacement of the mass and obtain clear sinusoidal oscillations, but the proposed configuration did not work.

Finally, a solution was found which involved a further modification of the mass shape. The mass was linked with a 6-mm-diameter threaded rod to a cylindrical magnet of force $15 \mathrm{~kg}$-pull, $20 \mathrm{~mm}$ long, $20 \mathrm{~mm}$ outer diameter, $6 \mathrm{~mm}$ inner diameter as shown in Fig. 3 so that this magnet could be placed inside the coil (approximately in the middle) of diameter $50 \mathrm{~mm}$ and length $50 \mathrm{~mm}$. The magnetic field is constant inside the coil on its axis if the magnet oscillates around the centre. The inner diameter of the coil is close to the diameter of the cylindrical magnet to improve the actuation surface between the varying field of the coil and the fixed field of the magnet and limit the loss of the field in the air.

Alternative methods of generating direct excitation on the mass were also explored. One of the considered ideas was to connect a linear DC servomotor directly to the mass. The other one was to connect the mass to a small shaker with a rod. Although the moving parts of the shaker used were six times lighter than the mass, during experiments it appeared that the behaviour of the system was dominated by the motion of the shaker 
Fig. 2 (a) Coil of $160 \mathrm{~mm}$ diameter and $160 \mathrm{~mm}$ length with $5 \mathrm{~kg}$ steel core and (b) mass assembly with different types of inserts tested: (1) mass covers; (2) steel insert; (3) 3D printed plastic perforated insert (ABS); (4) 3D printed plastic insert (ABS) with a cavity to add a square aluminium mass; and (5) 3D printed plastic insert with two cavities (a)

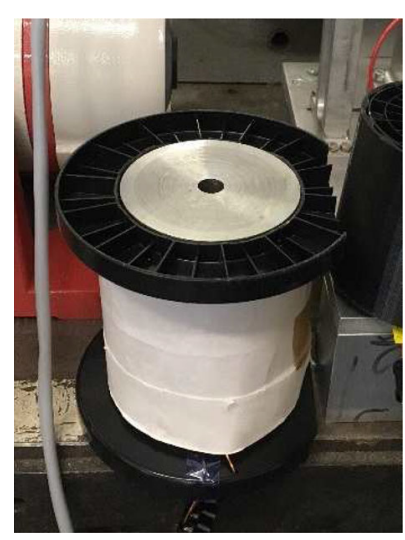

(b)

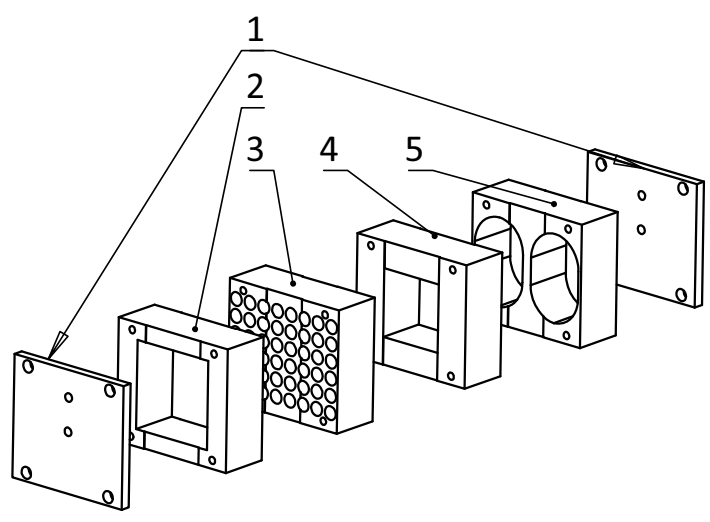

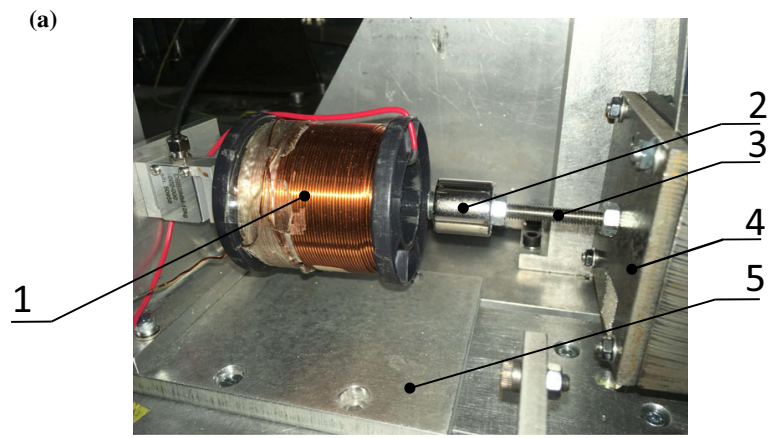

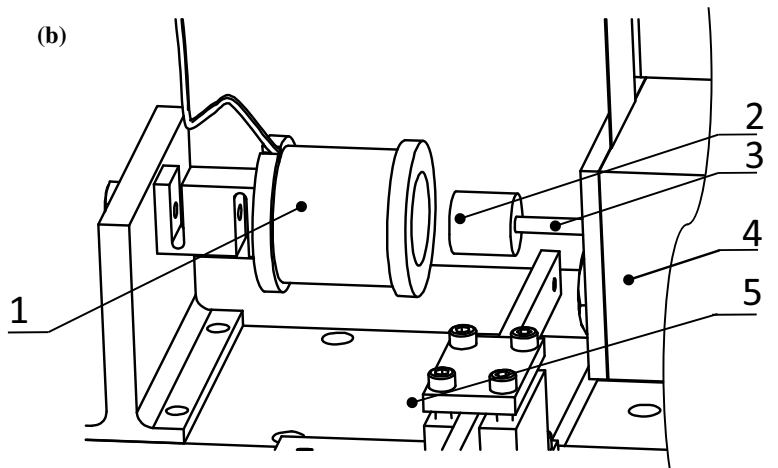

Fig. 3 View of the cylindrical magnet of strength $15 \mathrm{~kg}$-pull outside the coil of 470 turns of copper wire of $50 \mathrm{~mm}$ diameter and $50 \mathrm{~mm}$ length: (a) photograph; (b) schematic; with (1) coil; (2) magnet; (3) rod; (4) mass; and (5) support plate

and did not generate the expected dynamics of the oscillator.

Finally, the best experimental configuration was found based on the optimal reactivity of the mass on the variation in the magnetic field while keeping the natural dynamical behaviour of the oscillator. For the configuration shown in Fig. 3, the chosen actuation process allows to drive a relatively heavy mass achieving lower level of noise, and therefore, further experiments were undertaken using steel mass.

\subsubsection{Structural support}

The designed impact oscillator aims to study dynamics occurring during impacts, and it is essential to have an accurate measurement of the instant when impact occurs and the time length, when the mass is in contact with the secondary beam. The originally recorded experimental signals generated using the rig shown in Fig. 1 were characterised by a noise with very low amplitude and high frequency. This noise, even very small, was confusing the experimental results and the supporting structure had to be reinforced. Therefore, it was decided to strengthen the structural support by adding two extra columns symmetrically placed around the main column and fixing a triangular plate behind the main column as shown in Fig. 4. This extra reinforcement was designed to reduce the unwanted structural vibration when impacts occur.

The comparison of the results obtained using the originally designed rig and the improved one is shown in Fig. 5. Harmonic excitation force of the same frequency $4.5 \mathrm{~Hz}$ and the same amplitude of $0.9 \mathrm{~N}$ was applied, and the recorded signals from the force transducer are shown in Fig. 5a and b. The comparison of the fast Fourier transform of the acceleration of the base is presented in Fig. 5c for the original design (see Fig. 5e) and in Fig. 5d for the improved design (Fig. 5f). It can be seen that the noise at frequencies around $100 \mathrm{~Hz}$ 
Fig. 4 Schematic of the modified rig with the new parts marked in grey: (1) base plate; (2) main support column; (3) square pillar; (4) leaf spring; (5) mass; (6) adjustable bracket; (7) secondary support impact beams; (8) bracket plate; (9) coil; (10) rigid plate; (11) square pillar; (12) probe fixture; (13) side column; (14) side column; (15) and base legs

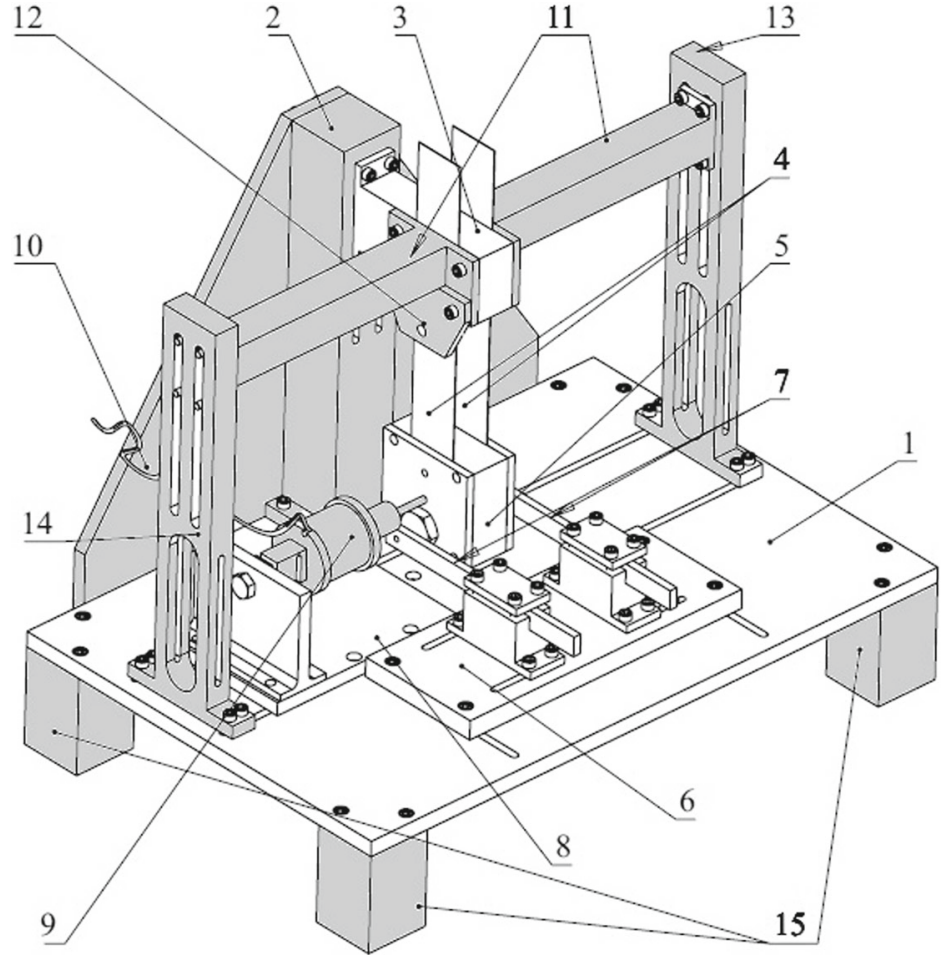

is considerably attenuated. There is a second range of frequencies around $300 \mathrm{~Hz}$ appearing in both cases.

The modification to the original design reduced the recorded noise significantly so that it becomes possible to recognise the time and the length of the impact. The noise occurring at higher frequencies is attenuated by a low-pass filter (with a cutting frequency fixed at $200 \mathrm{~Hz}$ ) at an acceptable range for the study of impacts.

\subsubsection{Instrumentation and control of magnetic force}

To enhance the monitoring of the system behaviour, a new force transducer and an accelerometer attached to the impact beam were added to the modified rig and the locations of all sensors are shown in Fig. 6. From three sensors described in Sect. 2.2, two sense the motion of the mass (an eddy current probe presented in Fig. 6a and an accelerometer attached to the mass shown in Fig. 6e) and one monitors the base behaviour (an accelerometer attached to the support plate as shown in Fig. 6c). In addition, a load cell shown in Fig. 6d and an impact beam accelerometer shown in Fig. 6e have been introduced in the new rig. This $S$-beam load cell was fitted between the coil frame and the coil, and it records the reaction force resulting from the interactions of the magnetic field with the magnet linked to the mass. The load cell is necessary to evaluate the force acting on the mass and can be used to control it or to characterise the coupling between the magnetic field and this force.

The recorded reaction force can vary considerably near the resonant frequency. However, most of the time, amplitude of the force acting on the mass needs to remain constant during specific set of experiments, and therefore, it needs to be controlled. To implement such control, a Proportional Integral Derivative (PID) controller routine has been created in LabVIEW which allows to vary automatically the current in the coil in order to keep the force amplitude constant. The expression of the control function is:

$F(t)=k_{p} i(t)+k_{i} \int_{t_{s}}^{t} i\left(t^{\prime}\right) \mathrm{d} t^{\prime}+k_{d} \frac{\mathrm{d} i(t)}{\mathrm{d} t}$,

where $F(t)$ and $i(t)$ are, respectively, the force and current signals, $t_{s}$ is the waiting time before applying the control and the gain values $k_{p}, k_{i}$ and $k_{d}$ depend on the frequency of the force signal and can be modified manually by the user in the LabVIEW interface. The controller runs until reaching the target value of force amplitude, and the data acquisition starts when the force amplitude is steady after a waiting time of length defined by the user. LabVIEW drives the vari- 
(a)

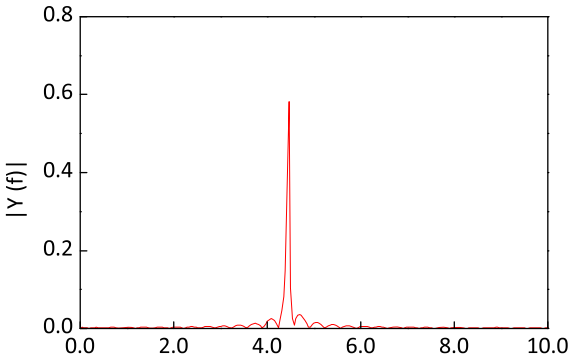

(c)

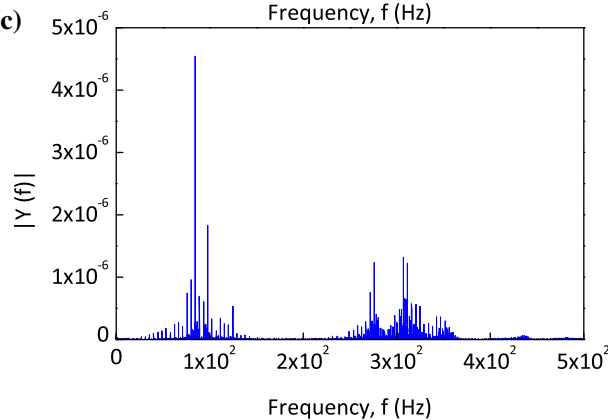

(e)

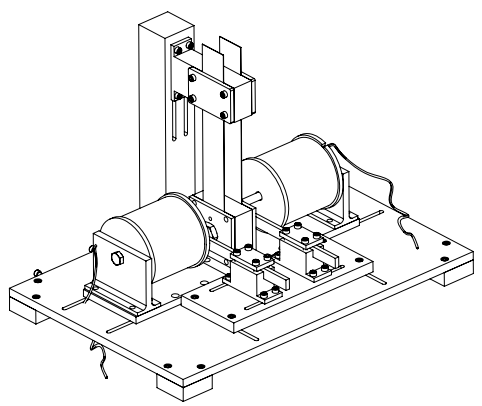

Fig. 5 (a) FFT of the input signal of the coil at an excitation frequency of $4.5 \mathrm{~Hz}$; (b) excitation signal of amplitude $0.9 \mathrm{~N}$; (c) FFT of the acceleration of the base for the original design; and (d) FFT of the acceleration of the base for the improved design.

able current in the coil and records data at the mean time. The current signal from LabVIEW needs to be amplified using two DC power supplies to obtain a current between 0 and $10 \mathrm{~A}$.

\section{Calibration and identification of the rig parameters}

To facilitate the comparison between the experimental and theoretical results, a systematic evaluation of the experimental parameters has been carried out. The installed eddy current probe was calibrated first, and then, the free vibration tests were conducted to find (b)

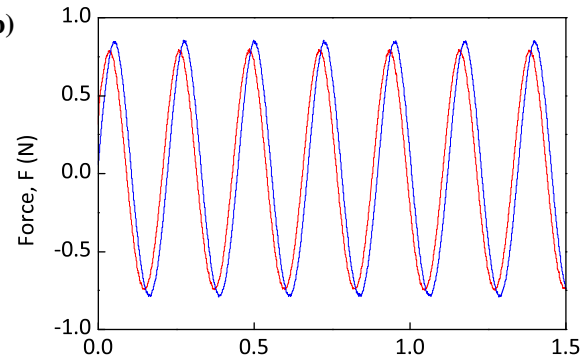

(d)

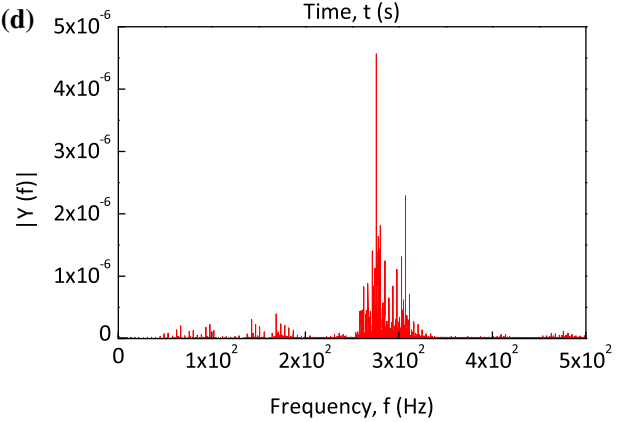

(f)

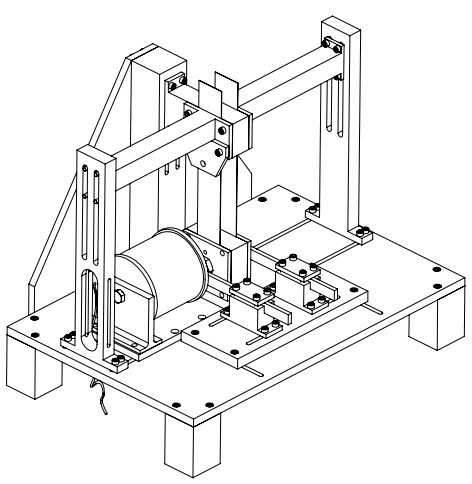

Blue colour marks the results obtained using the original rig and the results from the reinforced structure are in red; (e) original rig and (f) modified design. (Colour figure online)

out the natural frequency of the system and to estimate stiffness of the leaf springs and structural damping. It should be noted that when any changes are made to the length of leaf spring, location of the eddy current probe, or to the position of the mass, the same parameters identification process needs to be repeated.

\subsection{Eddy current probe calibration}

The eddy current probe situated on the top of the leaf springs records a variation in voltage resulting from the displacement of the leaf springs. The displacement on top of the leaf springs can be considered as being 
(a)

(d)
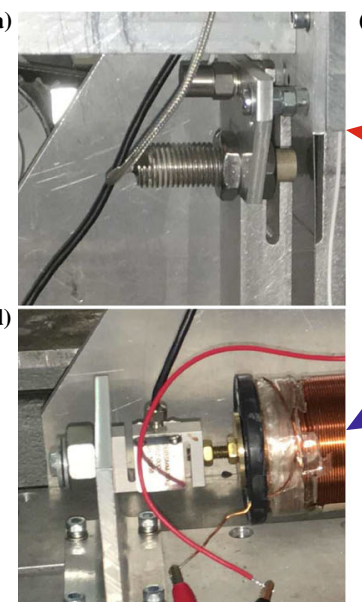

(b)

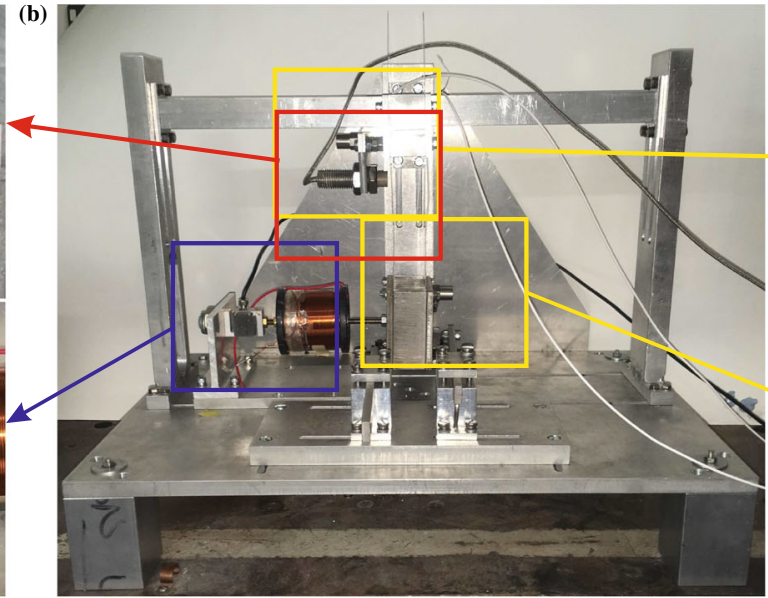

(c)

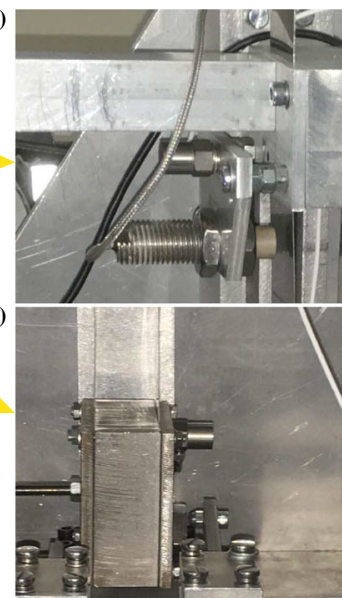

Fig. 6 Location of the sensors: (a) eddy current probe (red); (b) photograph of the whole rig; (c) accelerometer for the frame (yellow); (d) load cell (blue); and (e) accelerometer for the mass (yellow). (Colour figure online)

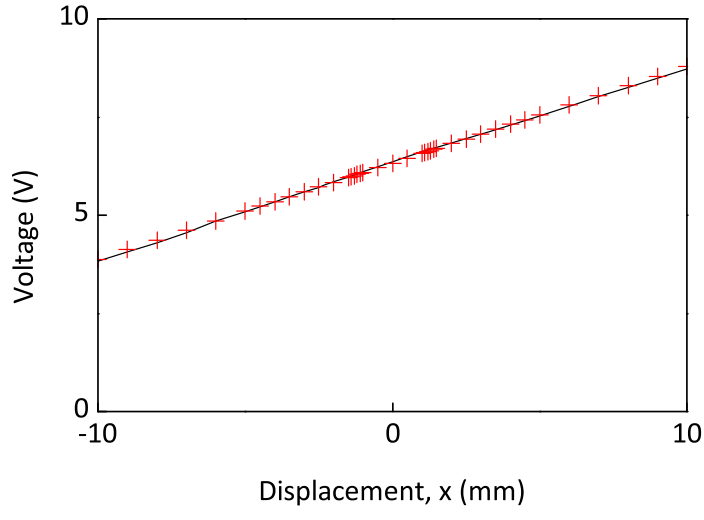

Fig. 7 Calibration results for the eddy current probe showing a linear relationship between the mass displacement and voltage. (Colour figure online)

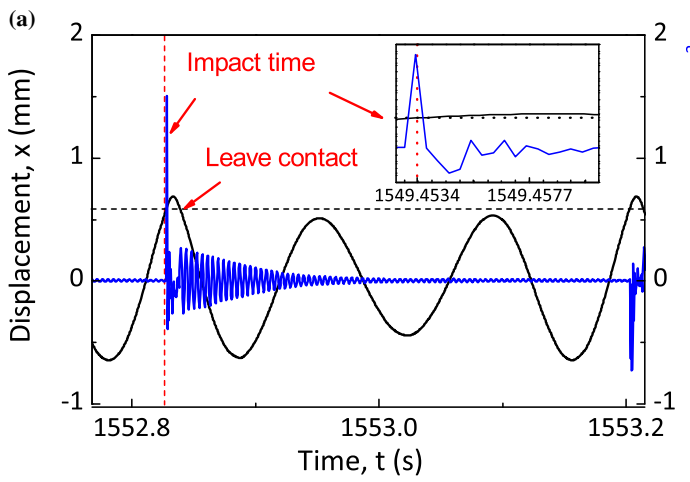

Fig. 8 Impact detection. Black dashed line marks the gap $g$, and dashed red lines indicate the time of impact. (a) Mass displacement and impact beam acceleration over time. The impact is identified as a peak in the beam acceleration signal, while the contact with the mass is lost when the signal becomes a damped proportional to the displacement of the mass at the bottom if the mass only oscillates horizontally in a small range around the equilibrium position. Inserts of known width are added between the mass and a fixed support, and the corresponding value of voltage of the eddy current probe is recorded ten times for inserts added on each side of the mass. Figure 7 presents the results of the calibration conducted for the leaf spring length of $105 \mathrm{~mm}$. As can be seen from this figure, there is a linear relationship between the recoded voltage from the eddy current probe and measured displacement of the mass. It is expressed as Diplacement $=($ Voltage -6.3298$) / 0.24552$.

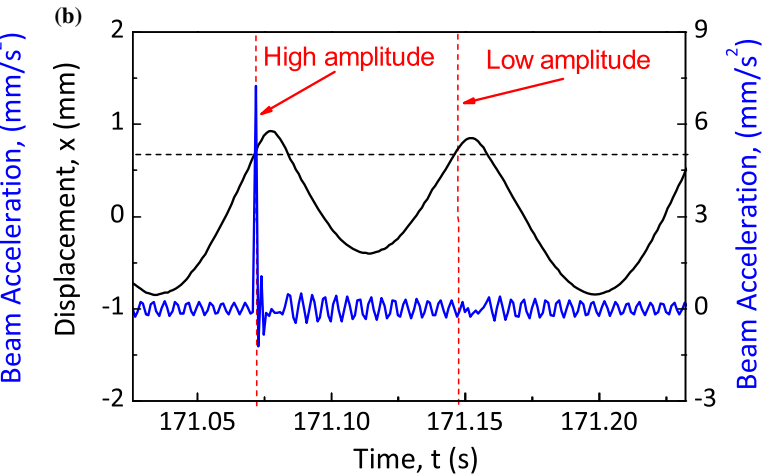

sinusoidal function. (b) A difference between a high-amplitude impact for which there is a large peak and low-amplitude impact where there is only a small disturbance in the acceleration signal. (Colour figure online) 


\subsection{Gap and the instant of impact}

The accelerometer placed on the impact beam is used to obtain the precise time of impact and the gap $g$ between the mass and impact beam. To improve the precision in setting the impact gap $g$, a screw was added to the tip of each impact beam. Impact start time was evaluated by identifying the first peak in the beam acceleration caused by a contact with the mass. Figure 8 shows the identification scheme for which the estimated errors in impact timing and the impact locations are $5 \times 10^{-5} \mathrm{~s}$ and $0.05 \mathrm{~mm}$, respectively. The moment when the mass lose contact with the beam can also be identified by the change of the impact beam acceleration signal to a damped sinusoidal function. These precise measurements can identify grazing incidences and help tune up the experimental rig to a desired behaviour. The impact beam acceleration signal also provides a way to qualitatively identify high and low amplitude beam acceleration impacts.

\subsection{Coefficient of damping and stiffness}

To determine the coefficients of damping and stiffness of the leaf springs, free vibration texts were performed. The sample of the recorded displacement of the mass is shown in Fig. 9a and b. The tests were repeated ten times, and the results were averaged to obtain the period of the free vibrations and the natural frequency. The

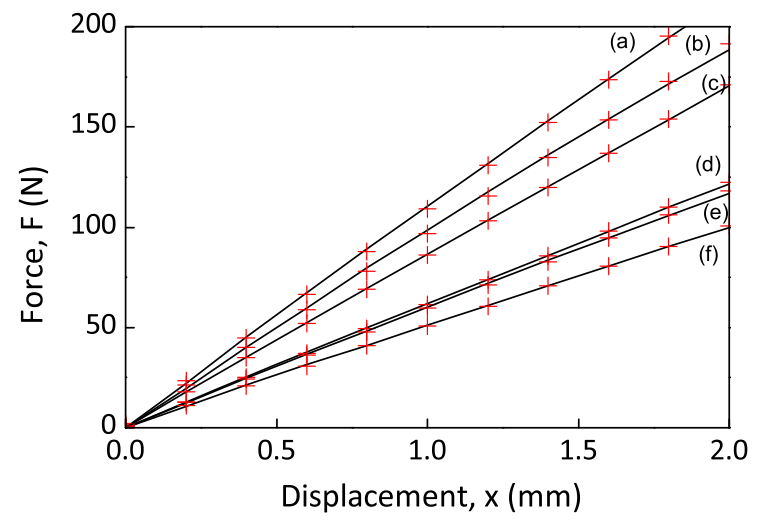

Fig. 10 Experimentally determined stiffness of secondary beam for (a) cross section $6 \times 15 \mathrm{~mm}^{2}$, length $95 \mathrm{~mm}$; (b) cross section $6 \times 15 \mathrm{~mm}^{2}$, length $100 \mathrm{~mm}$; (c) cross section $6 \times 15 \mathrm{~mm}^{2}$, length $105 \mathrm{~mm}$; (d) cross section $6 \times 5 \mathrm{~mm}^{2}$, length $95 \mathrm{~mm}$; (e) cross section $6 \times 5 \mathrm{~mm}^{2}$, length $100 \mathrm{~mm}$; and (f) cross section $6 \times$ $5 \mathrm{~mm}^{2}$, length $105 \mathrm{~mm}$. (Colour figure online)

Fast Fourier Transform of the displacement signal was also calculated, and the results are shown in Fig. 9c. The natural frequency was obtained as $f_{0}=9.3 \mathrm{~Hz}$ $\left(\omega_{0}=2 \pi f_{0}=58.3 \mathrm{rad} / \mathrm{s}\right)$, and the period of free vibrations was recorded as $T=0.11 \mathrm{~s}$. The logarithmic decrement method was used to determine the damping ratio, and the logarithm of maxima of displacement amplitude is presented in Fig. 9d. The damping ratio was obtained as $\zeta=0.00167$. The stiffness of the leaf spring was calculated using the natural frequency of the system as $k=M \omega_{0}^{2}=4.499 \mathrm{~N} / \mathrm{mm}$.
Fig. 9 Free vibration tests: (a) time history of the mass displacement after a small perturbation; (b) zoom of the time history where the maxima are marked by red cross; (c) amplitude spectrum of mass displacement; and (d) logarithm of displacement maxima. (Colour figure online)
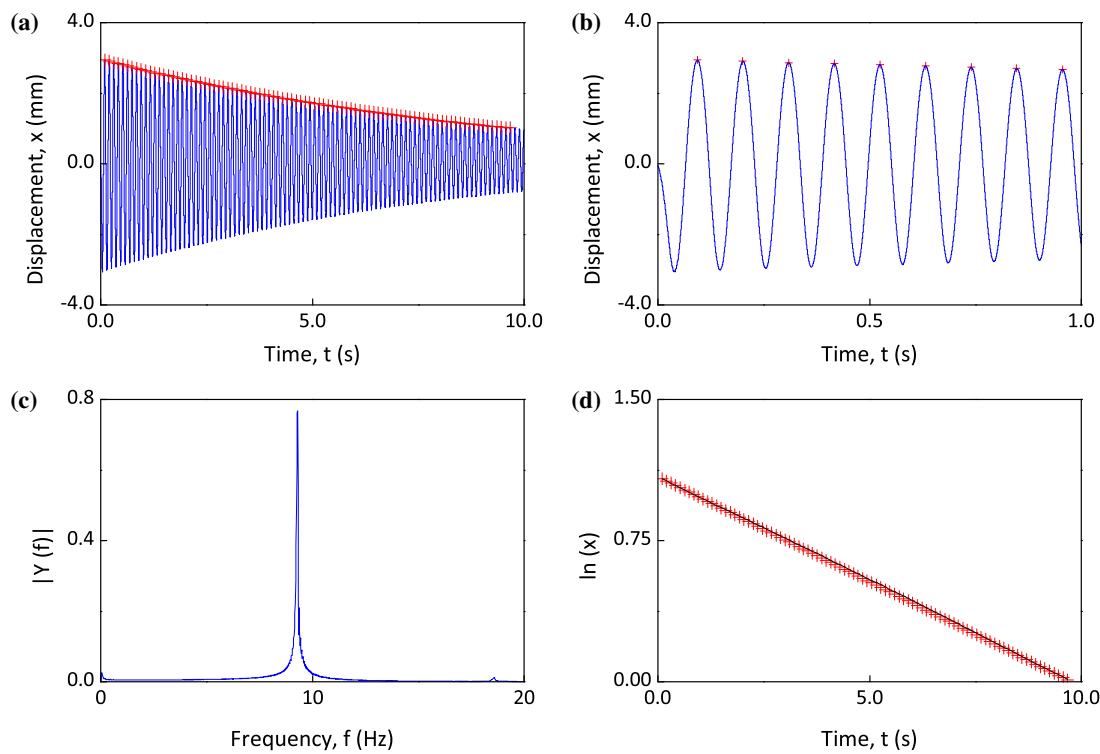

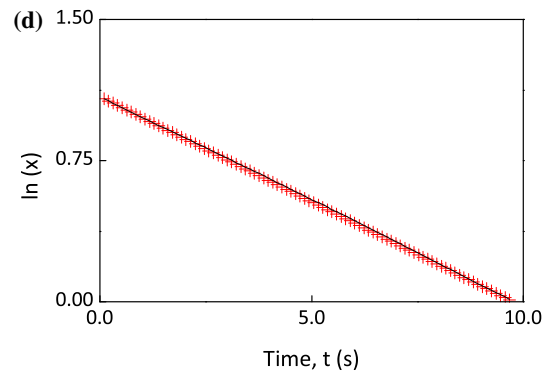


Table 1 Experimental parameter values for the new impact oscillator

\begin{tabular}{lll}
\hline Parameter & Value & Comments \\
\hline$m$ & $1.3 \mathrm{~kg}$ & Sensors weight $0.025 \mathrm{~kg}$ extra \\
Coil turns & $470 \mathrm{turns}$ & Coil of $50 \mathrm{~mm}$ diameter and $50 \mathrm{~mm}$ length \\
$I$ & $0-10 \mathrm{~A}$ & \\
$k_{1}$ & $4.499 \mathrm{~N} / \mathrm{mm}$ & Leaf spring length $L=115 \mathrm{~mm}$ \\
$k_{2}$ & $60.8 \mathrm{~N} / \mathrm{mm}$ & Impact beam section $6 \times 5 \mathrm{~mm}^{2}$, length $L=95 \mathrm{~mm}$ \\
& $58.5 \mathrm{~N} / \mathrm{mm}$ & Impact beam section $6 \times 5 \mathrm{~mm}^{2}$, length $L=100 \mathrm{~mm}$ \\
& $49.7 \mathrm{~N} / \mathrm{mm}$ & Impact beam section $6 \times 5 \mathrm{~mm}^{2}$, length $L=105 \mathrm{~mm}$ \\
& $107.4 \mathrm{~N} / \mathrm{mm}$ & Impact beam section $6 \times 15 \mathrm{~mm}^{2}$, length $L=95 \mathrm{~mm}$ \\
& $94.6 \mathrm{~N} / \mathrm{mm}$ & Impact beam section $6 \times 15 \mathrm{~mm}^{2}$, length $L=100 \mathrm{~mm}$ \\
& $85 \mathrm{~N} / \mathrm{mm}$ & Impact beam section $6 \times 15 \mathrm{~mm}^{2}$, length $L=105 \mathrm{~mm}$ \\
& $9.27 \mathrm{~Hz}$ & Leaf spring length $L=115 \mathrm{~mm}^{2}$ \\
& &
\end{tabular}

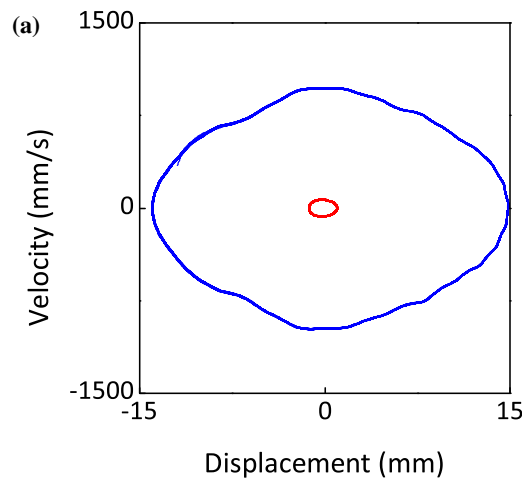

(b)

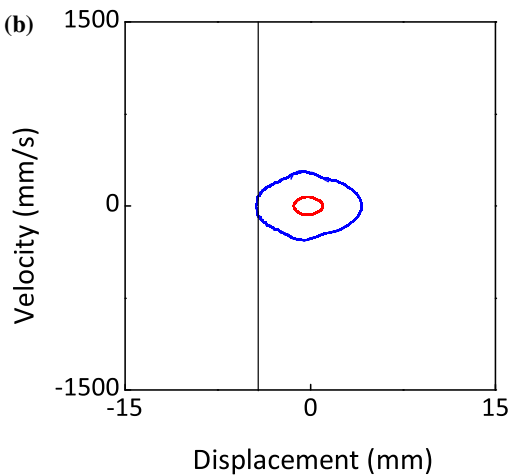

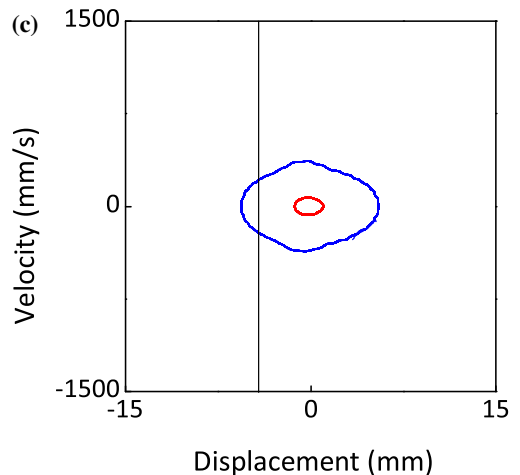

Fig. 11 Co-existing attractors for an harmonic forcing of amplitude $0.5 \mathrm{~N}$ and frequency $9.35 \mathrm{~Hz}$ for (a) non-impacting system; (b) system impacting a beam of section $6 \times 15 \mathrm{~mm}^{2}$ with a gap of

The parameters of the impact support beam could be studied following the same process, but its dimension is small for adding a sensor and record the vibration. Therefore, instead the static tests were conducted where the value of the stiffness was determined by statically deflecting the beam with a press and recording the load necessary to reach a certain displacement. The results of these tests are shown in Fig. 10 where force versus displacement curves are presented for six different configurations of the impact beam. Two cross sections were investigated, specifically $6 \times 5 \mathrm{~mm}^{2}$ and $6 \times 15 \mathrm{~mm}^{2}$, and three different values of the beam length were considered of 95, 100 and $105 \mathrm{~mm}$. Static tests were repeated five times for each combination, and then, the averaged impact support beam stiffness was obtained. The experimental parameters including
$4 \mathrm{~mm}$ (boundary marked by a black line); and (c) system impacting a beam of section $6 \times 5 \mathrm{~mm}^{2}$ with a gap of $4 \mathrm{~mm}$. (Colour figure online)

all variations in the support beam stiffness are summarized in Table 1.

\section{Preliminary experimental results}

In this section, the initial experimental results are presented. The study has been performed for three system configurations: the oscillator without a constraint, for the oscillator with a high stiffness beam constraint and for the oscillator with a low stiffness beam constraint. The set-up of the rig is the same for each case, and a beam is placed at a distance (gap) of $4 \mathrm{~mm}$ from the mass equilibrium position. The system parameters were readjusted slightly resulting in linear natural frequency around $9.1 \mathrm{~Hz}$ which is different from the value 
Fig. 12 Non-impacting response of an unconstrained system for an harmonic forcing at frequency $9.35 \mathrm{~Hz}$ : (a) input voltage from the generator; (b) coil voltage; (c) reaction force; (d) acceleration of the base; (e) acceleration of the mass; and (f) displacement of the mass
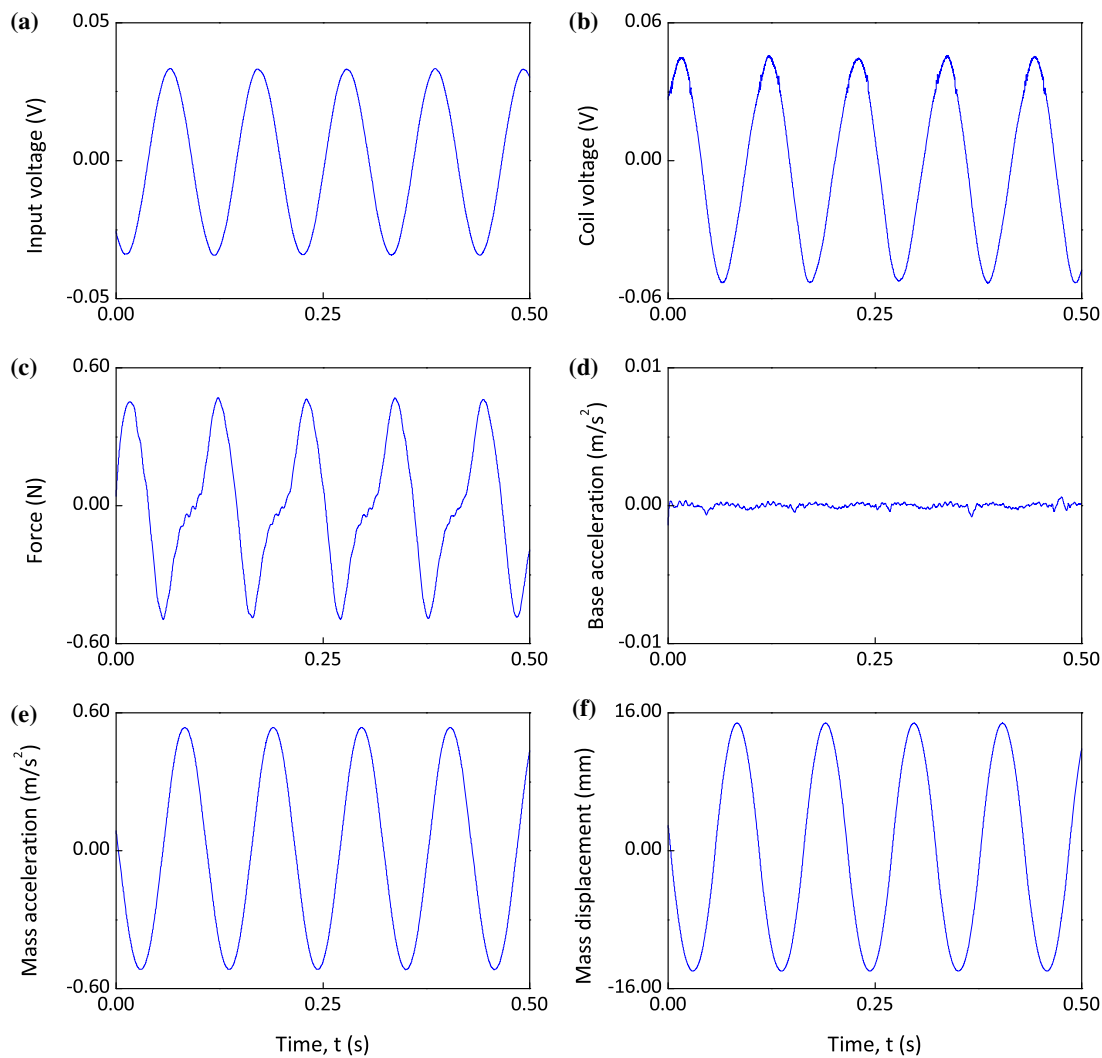

given in Table 1. A beam of larger cross section is used to investigate a harder impact scenario and the one of smaller cross section, more flexible, to consider softer impacts. The results are presented as the selected trajectories on the phase plane as shown in Fig. 11, time histories of the recorded signals as demonstrated in Figs. 12, 13, 14 and 15 and bifurcation diagrams in Fig. 16.

In Fig.11, trajectories on the phase plane are shown for three considered cases when the oscillator is excited by a harmonic force of amplitude of $0.5 \mathrm{~N}$ and frequency $9.35 \mathrm{~Hz}$ which is chosen to be close to the resonance frequency of the mechanical system.

Figure 11a demonstrates the response of the system without constraint, whereas Fig.11b shows the response of the system with an impact beam of section $6 \times 15 \mathrm{~mm}^{2}$ and Fig. $11 \mathrm{c}$ presents the response of the system with an impact beam of section $6 \times 5 \mathrm{~mm}^{2}$. As can be seen from this figure, in all three cases coexisting responses are observed where a low amplitude non-impacting orbit co-exists with a large amplitude orbit. In Fig.11a, this larger orbit has the highest amplitude which is reduced with the increase in impact beam stiffness. As can be seen in Figs.11b and c, higher level of noise caused by the impacts is observed in the system with stiffer beam which is presented in part (b).

Figures 12, 13, 14 and 15 present the scenarios shown in Fig. 11 in more details. In these figures, each recorded time history is shown from 0 to 0.5 second, but a waiting time of 30 seconds has been observed before recording the data to avoid the transient state. The harmonic voltage produced by a generator using LabVIEW is shown in parts (a) of these figures. It should be noted that this signal is recorded when a feedback loop is applied to control the force amplitude at the required level of $0.5 \mathrm{~N}$. Parts (b) show the measured voltage in the coil, and parts (c) present the reaction force measured by the sensor discussed previously. Parts (d) demonstrate the acceleration of the base, and finally, parts (d) and (f) show the acceleration and displacement of the mass, respectively.

The non-impacting system is considered first, and time histories of the signals recorded are shown for large amplitude orbit in Fig. 12 and small amplitude orbit in Fig. 13. The former one corresponds to blue orbit presented in Fig.11a, and the later one corresponds to the red orbit. In both cases, the acceleration of the 
Fig. 13 Co-existing non-impacting response of an unconstrained system for an harmonic forcing at frequency $9.35 \mathrm{~Hz}$ : (a) input voltage from the generator; (b) coil voltage; (c) reaction force; (d) acceleration of the base; (e) acceleration of the mass; and (f) displacement of the mass. (Colour figure online)

Fig. 14 Impacting response of the system constrained by the beam of section $6 \times 15 \mathrm{~mm}^{2}$ with a gap of $4 \mathrm{~mm}$ and excitation frequency of $9.35 \mathrm{~Hz}$ : (a) input voltage from the generator; (b) coil voltage; (c) reaction force; (d) acceleration of the base; (e) acceleration of the mass; and (f) displacement of the mass (The black line shows the impact boundary).

(Colour figure online)
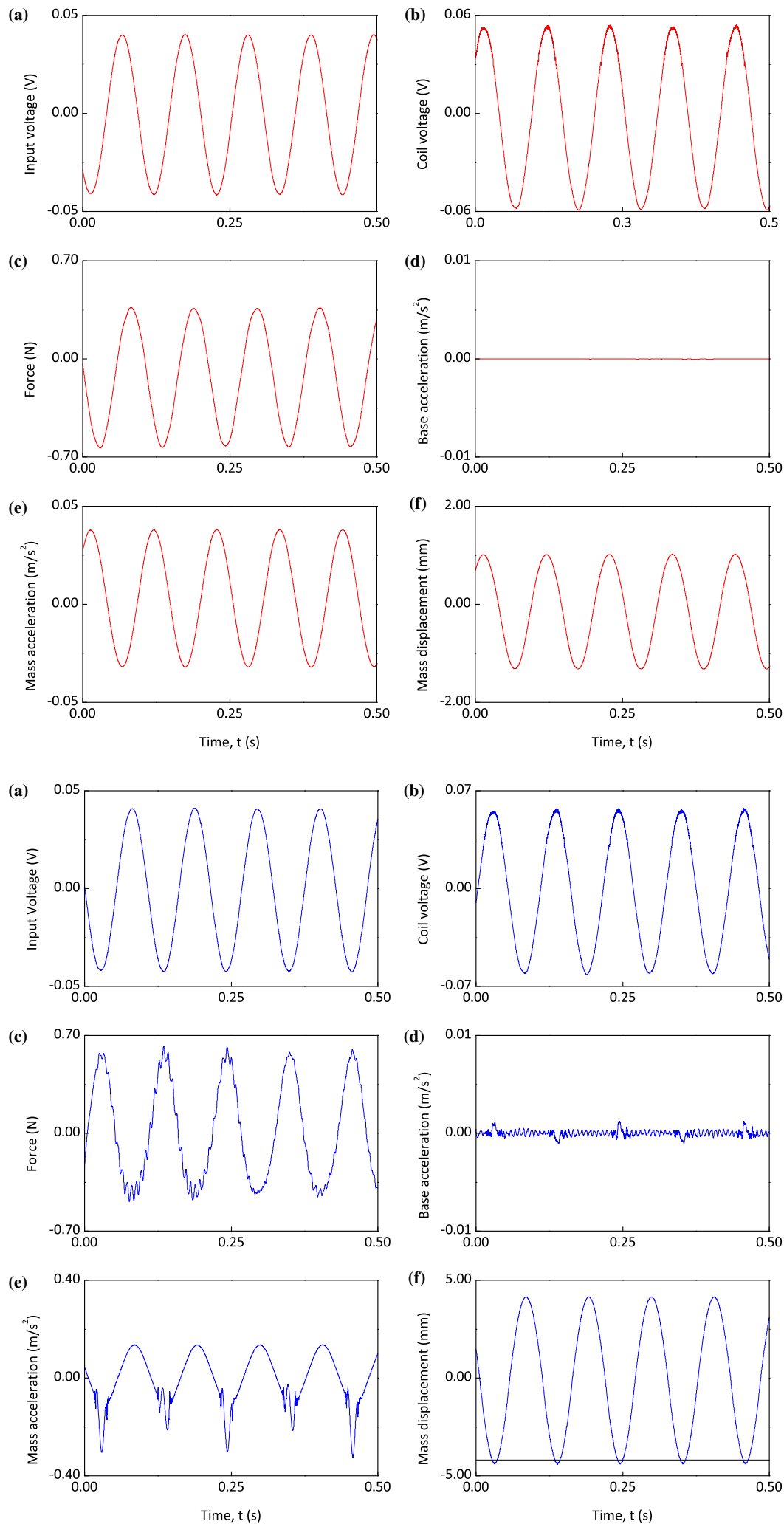
Fig. 15 Impacting response of the system constrained by the beam of section $6 \times 5 \mathrm{~mm}^{2}$ at a gap of $4 \mathrm{~mm}$ for an harmonic forcing at frequency $9.35 \mathrm{~Hz}$ : (a) input voltage from the generator; (b) coil voltage; (c) reaction force; (d) acceleration of the base; (e) acceleration of the mass; and (f) displacement of the mass (The black line shows the impact boundary)
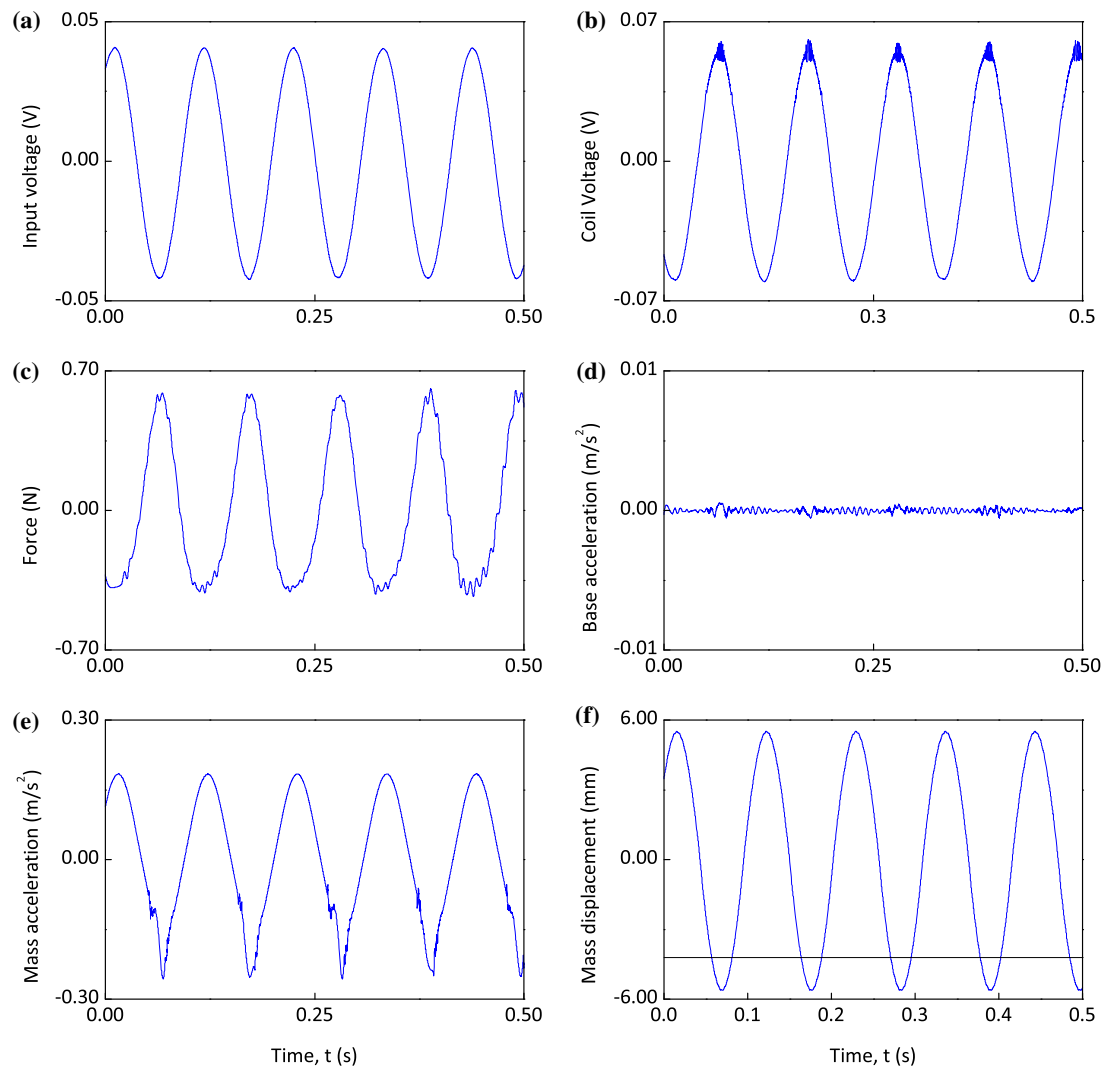

mass is periodic and the acceleration of the base is close to zero. The main difference is observed in the shape of the signal of the reaction force, which it is no longer harmonic for the higher amplitude response as shown in Fig. 12c.

Figure 14 presents time histories of the recorded signals of large amplitude orbit for the oscillator constrained by the beam of cross section $6 \times 15 \mathrm{~mm}^{2}$ which is introduced in Fig.11b. Here, the impact is clearly visible both in the mass displacement and acceleration signals in parts (e) and (f). Black horizontal line in Fig. 14f shows the location of the impact beam, and impacts occur when displacement crosses this line. In Fig. 14e, impacts are manifested by sharp peaks in acceleration signals, and width and height of these peaks reveal the duration and the strength of the impacts as previously discussed in [26]. As can be seen from Fig. 14d, the amplitude of the base acceleration is still low, but occurrences of the impacts can be identified by larger peaks in the signal. Finally, the presence of higher harmonics in the reaction force signal shown in Fig. 14c should be noted. For the small red amplitude orbit from Fig. 11b, time histories of the signals are very similar to those presented earlier in Fig. 13.

Finally, Fig. 15 depicts time histories of the recorded signals of the large amplitude orbit for the oscillator constrained by the beam of cross section $6 \times 15 \mathrm{~mm}^{2}$ which is introduced in Fig.11c. The same as in the previous case, impacts are easily identified on the mass acceleration and displacement trajectories. In this case of softer beam, they are also visible in the coil voltage signal in Fig. 15b. It is interesting to note that due to softer beam, the height of the impact related peaks is smaller in the base acceleration signal than it was observed in the case of harder beam.

The three cases shown in Fig. 11 are part of the bifurcation diagrams presented in Fig. 16 where the results for the system without constraint are demonstrated in Fig. 16a and b, for the system with the impact beam of section $6 \times 15 \mathrm{~mm}^{2}$ and the gap of $4 \mathrm{~mm}$ in Fig. $16 \mathrm{c}$ and $\mathrm{d}$, and for the system with the impact beam of section $6 \times 5 \mathrm{~mm}^{2}$ and the same gap in Fig. 16e and $\mathrm{f}$. These diagrams are constructed by increasing and decreasing the excitation frequency in the range $8.9-9.5 \mathrm{~Hz}$ for 
Fig. 16 Bifurcation diagrams in blue constructed by increasing the frequency values and in red for decreasing the frequency in the range 8.9-9.5 Hz. The black line marks the frequency chosen for the phase planes shown in Fig.11 (9.35 Hz) (a) and (b) The non-impacting system; (c) and (d) system with the impact beam of section $6 \times 15 \mathrm{~mm}^{2}$ and the gap of $4 \mathrm{~mm}$; and (e) and (f) system with the impact beam of section $6 \times 5 \mathrm{~mm}^{2}$ and the gap of $4 \mathrm{~mm}$. (Colour figure online)
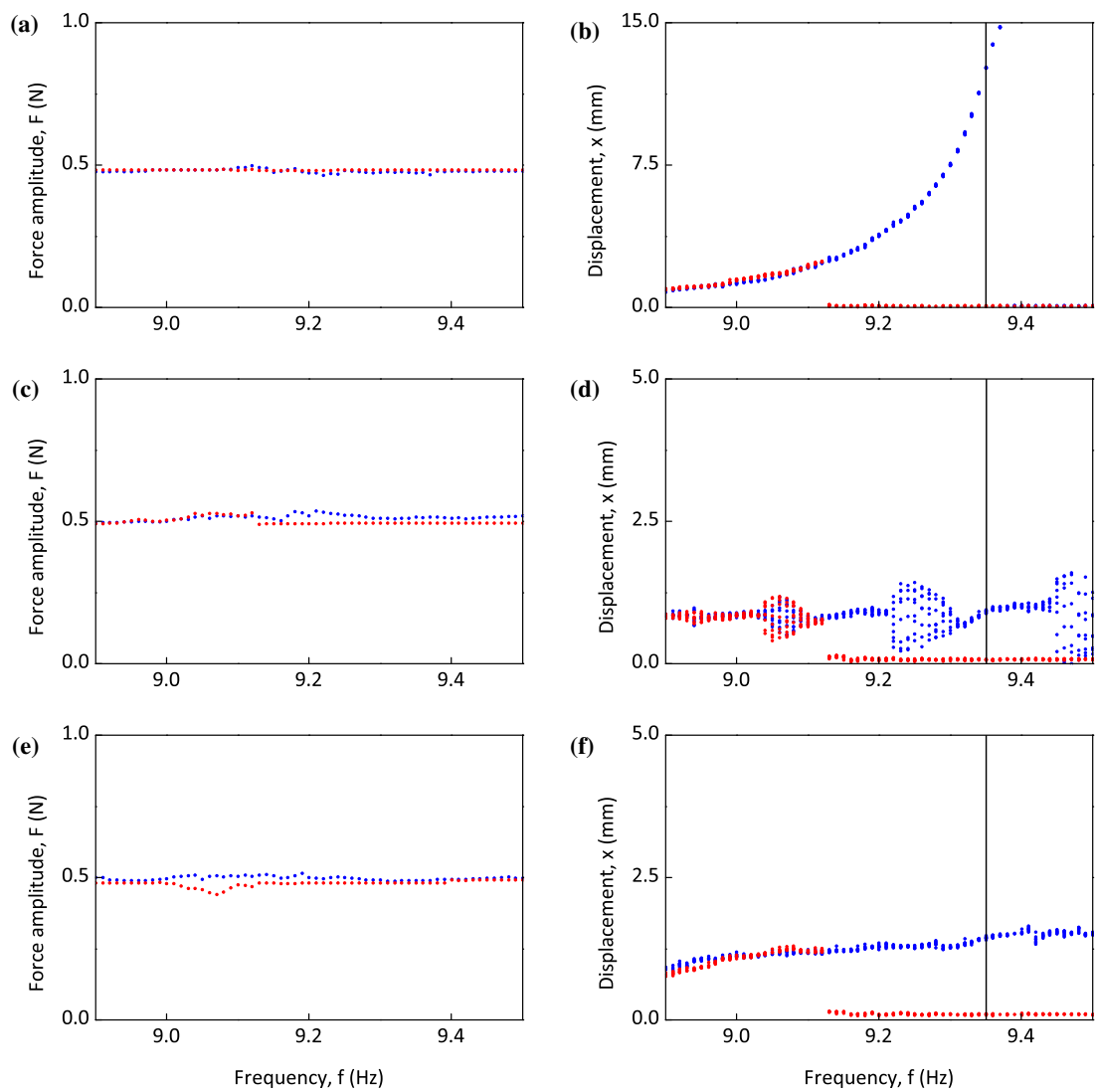

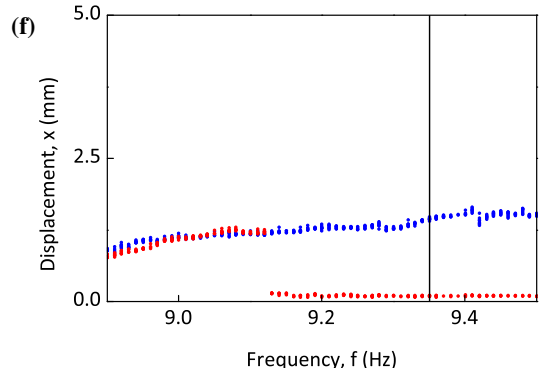

all three considered cases and shown in blue and red, respectively. Parts (a), (c) and (d) present the measured amplitude of the reaction force which was aimed to be kept at the level of 0.5 N. Parts (b), (d) and 9(f) show the values of the displacement as function of the frequency. As can be seen from this figure, the applied control was effective in keeping the amplitude of the force acting on the mass constant at $0.5 \mathrm{~N}$. Non-impacting linear responses were observed for all three system configurations below the first grazing incident. Above 9.13 $\mathrm{Hz}$, the co-existing solutions are recorded in all three cases. Our further investigation shows that for the system without constraint, those are caused by nonlinear behaviour of the leaf springs supporting the oscillator mass when the large mass displacement is observed close to the mechanical system resonance [39]. For the two cases where the impact beam is present, the nonlinearities are caused by the impacts alone as the displacement of the mass is restricted in this case. Low amplitude responses observed at higher frequencies and shown in red in Fig 16b, $d$ and $f$ are non-impacting linear responses demonstrated in Fig. 11. As can be seen from Fig. 16d, a mixture of impacting period-1 and chaotic responses is recorded for the configuration with the stiffer impact beam and the detailed bifurcation analysis of such impacting system will be a subject of further investigation to be presented in a separate publication.

\subsection{Final improvements}

Further modifications were introduced to reduce the nonlinearities related to the magnetic coupling. The iron rod connecting the magnet and the mass was replaced by a stainless steel one to eliminate any hysteretic behaviour due to induced magnetization. Also, a realignment of the mass and coil was performed to better position the magnet inside the coil. These processes altered slightly the leaf springs active length leading to slight variations on the rig parameters. The same identification procedure was performed to obtain the new parameters of the rig: leaf springs stiffness $k_{1}=4330 \mathrm{~N} / \mathrm{m}$ and linear damping ratio $\zeta=0.0018$. 
Fig. 17 Co-existing non-impacting response of an unconstrained system for an harmonic forcing at frequency $9.3 \mathrm{~Hz}$ : (a) input voltage from the generator; (b) coil voltage; (c) reaction force; (d) acceleration of the base; (e) acceleration of the mass; and (f) displacement of the mass
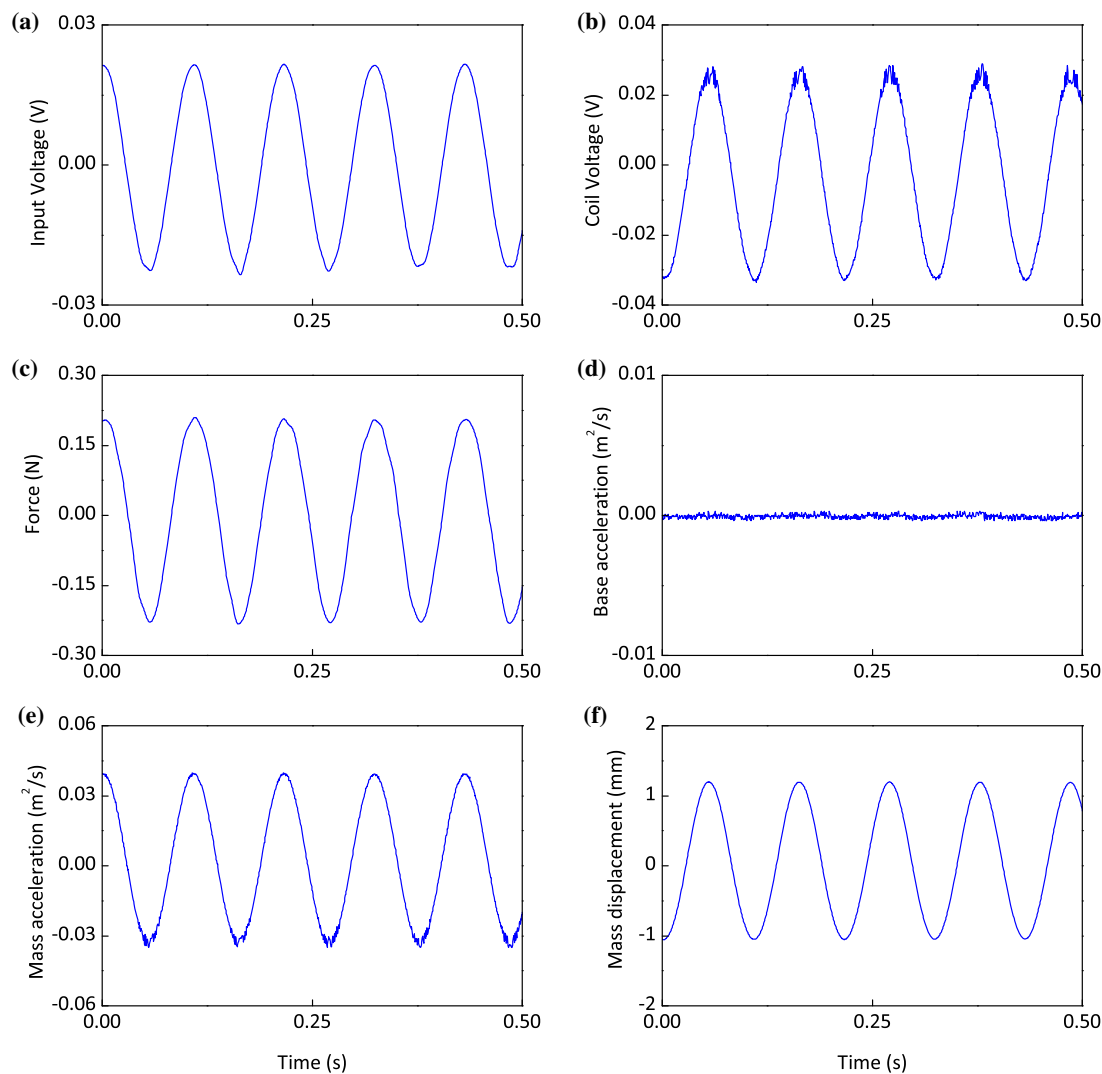

Figure 17 shows the detailed signals for the nonimpacting case at the frequency of $9.3 \mathrm{~Hz}$, near the new resonance frequency of $9.1 \mathrm{~Hz}$. There is little to none nonlinearities present in the reaction force signal in Fig. 17c or any excitation signals as shown in Figs 17a and $b$. Also, the noise on the force signal is reduced. These results show that the magnetic field coupling nonlinearities were reduced by the applied changes.

\section{Closing remarks}

The design evolution and the initial experimental results of the novel impact oscillator rig developed by the Centre of Applied Dynamics Research at the University of Aberdeen have been presented. In this rig, the excitation force is generated electromagnetically and it acts directly on the mass in contrast to the most of the similar rigs where the excitation passes through the structure. This significantly enhances the flexibility of the system control allowing to follow a particular orbit under parameter changes and(or) to observe subtle new phenomena. The oscillator has been designed considering the simplicity of manufacturing, the cost and the adaptability of the parameters to cover a wide range of experimental configurations.

The actuation process of the mass has been investigated, and several types of excitation methods have been compared. The final configuration employs one coil of copper where an alternating current generates magnetic field introducing excitation force on the mass. The current signal is adjusted and controlled with a LabVIEW program to obtain a desired excitation force. Impact beams are introduced to study impact phenomena in details, and careful considerations of the suitable operating range will be required to determine the system parameters where impact nonlinearities are separated from effects introduced by nonlinear behaviour of the leaf springs. In general, for large gaps and high forcing amplitudes combined nonlinear effects are expected in the system response; however, for low forcing amplitudes and small gaps the system can exhibit pure impact nonlinearities and can be used to study impact phenomena. The preliminary experimental results demonstrate a wide range of non- 
linear responses including various periodic orbits, coexistence of attractors and chaotic behaviour. The direct excitation applied to the mass allows to implement control to the system, and it can be used to provide nonharmonic excitations as well.

Acknowledgements Contributions of the technicians at the University of Aberdeen to the development of this experimental rig is gratefully acknowledged by the authors: in particular, Edward Stephen for creating the LabVIEW program controlling the excitation force parameter and recording the experimental data, Raymond Stephen for the electrical equipment provided, and the mechanical workshop for the improvements in the rig. The authors also acknowledge the support from and Chinese Scholarship Council (CSC Grant Number 11502161). A part of this project was funded by Coordenação de Aperfeiçoamento do Pessoal de Nível Superior (CAPES) under the Grant Number 88881.189487/2018-01.

\section{Compliance with ethical standards}

Conflict of interest The authors declare that they have no conflict of interest.

Open Access This article is distributed under the terms of the Creative Commons Attribution 4.0 International License (http:// creativecommons.org/licenses/by/4.0/), which permits unrestricted use, distribution, and reproduction in any medium, provided you give appropriate credit to the original author(s) and the source, provide a link to the Creative Commons license, and indicate if changes were made.

\section{References}

1. Melamed, Y., Kiselev, A., Gelfgat, M., Dreesen, D., Blacic, J.: Hydraulic hammer drilling technology: developments and capabilities. J. Energy Resour. Technol. 122(1), 1-7 (1999)

2. Wiercigroch, M.: Resonance enhanced drilling: method and apparatus. patent no. WO2007141550. (2007)

3. Pavlovskaia, E., Hendry, D.C., Wiercigroch, M.: Modelling of high frequency vibro-impact drilling. Int. J. Mech. Sci. 91, 110-119 (2015)

4. Liao, M., Ing, J., Sayah, M., Wiercigroch, M.: Dynamic method of stiffness identification in impacting systems for percussive drilling applications. Mech. Syst. Signal Process 80, 224-244 (2016)

5. Jeffcott, H.H.: XXVII. The lateral vibration of loaded shafts in the neighbourhood of a whirling speed. The effect of want of balance. Philos. Mag. Ser. 6 37(219), 304-314 (1919)

6. Karpenko, E.V., Wiercigroch, M., Cartmell, M.P.: Regular and chaotic dynamics of a discontinuously nonlinear rotor system. Chaos Solitons Fractals 13(6), 1231-1242 (2002)

7. Karpenko, E.V., Pavlovskaia, E., Wiercigroch, M.: Bifurcation analysis of a preloaded jeffcott rotor. Chaos Solitons Fractals 15(2), 407-416 (2003)
8. Pavlovskaia, E.E., Karpenko, E.V., Wiercigroch, M.: Nonlinear dynamic interactions of a jeffcott rotor with preloaded snubber ring. J. Sound Vib. 276(1), 361-379 (2004)

9. Karpenko, E.V., Pavlovskaia, Wiercigroch, E.E., Neilson, R.D.: Experimental verification of jeffcott rotor model with preloaded snubber ring. J. Sound Vib. 298(4), 907-917 (2006)

10. Thompson, J.M.T., Bokaian, A.R., Ghaffari, R.: Subharmonic and chaotic motions of compliant offshore structures and articulated mooring towers. J. Energy Resour. Technol. 106(2), 191-198 (1984)

11. Peterka, F.: Laws of impact motion of mechanical systems with one degree of freedom. i theoretical analysis of nmultiple (1/n)-impact motions. Acta Technica Ceskoslovenska Akademie Ved 19, 462-473 (1974)

12. Peterka, F.: Laws of impact motion of mechanical systems with one degree of freedom. part II-results of analogue computer modelling of the motion. Acta Technica Ceskoslovenska Akademie Ved 19, 569-58 (1974)

13. Whiston, G.S.: Global dynamics of a vibro-impacting linear oscillator. J. Sound Vib. 118(3), 395-424 (1987)

14. Whiston, G.S.: Singularities in vibro-impact dynamics. J. Sound Vib. 152(3), 427-460 (1992)

15. Nordmark, A.B.: Non-periodic motion caused by grazing incidence in an impact oscillator. J. Sound Vib. 145(2), 279297 (1991)

16. Peterka, F., Vacik, J.: Transition to chaotic motion in mechanical systems with impacts. J. Sound Vib. 154(1), 95115 (1992)

17. Peterka, F.: Bifurcations and transition phenomena in an impact oscillator. Chaos Solitons Fractals 7(10), 1635-1647 (1996)

18. Peterka, F.: Behaviour of impact oscillator with soft and preloaded stop. Chaos Solitons Fractals 18(1), 79-88 (2003)

19. Pust, L., Peterka, F.: Impact oscillator with hertz's model of contact. Meccanica 38(1), 99-116 (2003)

20. Peterka, F., Kotera, T., Cipera, S.: Explanation of appearance and characteristics of intermittency chaos of the impact oscillator. Chaos Solitons Fractals 19(5), 1251-1259 (2004)

21. Moon, F.C., Shaw, S.W.: Chaotic vibrations of a beam with non-linear boundary conditions. Int. J. Non-Linear Mech. 18(6), 465-477 (1983)

22. Shaw, S.W.: Forced vibrations of a beam with one-sided amplitude constraint: theory and experiment. J. Sound Vib. 99, 199-212 (1985)

23. Sin, V.W.T., Wiercigroch, M.: A symmetrically piecewise linear oscillator: design and measurement. Proc. Inst. Mech. Eng. Part C J. Mech. Eng. Sci. 213(3), 241-249 (1999)

24. Wiercigroch, M., Sin, V.W.T.: Experimental study of a symmetrical piecewise base-excited oscillator. J. Appl. Mech. Trans. ASME 65(3), 657-663 (1998)

25. Ing, J., Pavlovskaia, E., Wiercigroch, M.: Dynamics of a nearly symmetrical piecewise linear oscillator close to grazing incidence: modeling and experimental verification. Nonlinear Dyn. 46(3), 225-238 (2006)

26. Ing, J., Pavlovskaia, E., Wiercigroch, M., Banerjee, S.: Experimental study of impact oscillator with one-sided elastic constraint. Philos. Trans. R. Soc. Lond. A Math. Phys. Eng. Sci. 366(1866), 679-705 (2008)

27. Ing, J., Pavlovskaia, E., Wiercigroch, M., Banerjee, S.: Bifurcation analysis of an impact oscillator with a one-sided 
elastic constraint near grazing. Phys. D Nonlinear Phenom. 239(6), 312-321 (2010)

28. George, C., Virgin, L.N., Witelski, T.: Experimental study of regular and chaotic transients in a non-smooth system. Int. J. Non-Linear Mech. 81, 55-64 (2016)

29. Paez Chavez, J., Wiercigroch, M.: Bifurcation analysis of periodic orbits of a non-smooth jeffcott rotor model. Commun. Nonlinear Sci. Numer. Simul. 18(9), 2571-2580 (2013)

30. Liu, Y., Wiercigroch, M., Ing, J., Pavlovskaia, E.: Intermittent control of coexisting attractors. Philos. Trans. R. Soc. Lond. A Math. Phys. Eng. Sci. 371, 2013 (1993)

31. Mason, J.F., Piiroinen, P.T., Wilson, R.E., Homer, M.E.: Basins of attraction in nonsmooth models of gear rattle. Int. J. Bifurc. Chaos 19(1), 203-224 (2009)

32. Kuribayashi, K., Tsuchiya, K., You, Zh, Tomus, D., Umemoto, M., Ito, T., Sasaki, M.: Self-deployable origami stent grafts as a biomedical application of ni-rich TiNi shape memory alloy foil. Mater. Sci. Eng. A 419(1), 131-137 (2006)

33. Salerno, M., Zhang, K., Menciassi, A., Dai, J.S.: A novel 4-DOF origami grasper with an SMA-actuation system for minimally invasive surgery. IEEE Trans. Robot. 32(3), 484498 (2016)
34. Barbosa, W.O.V., De Paula, A.S., Savi, M.A., Inman, D.J.: Chaos control applied to piezoelectric vibration-based energy harvesting systems. Eur. Phys. J. Spec. Topics 224(14), 2787-2801 (2015)

35. Souza de Paula, A., Savi, M.A.: Comparative analysis of chaos control methods: a mechanical system case study. Int. J. Non-Linear Mech. 46(8), 1076-1089 (2011)

36. Costa, D.A., Savi, M.A., De Paula, A.S., Bernardini, D.: Chaos control of a shape memory alloy structure using thermal constrained actuation. Int. J. Non-Linear Mech. 111, 106-118 (2019)

37. Pisarchik, A.N., Feudel, U.: Control of multistability. Phys. Rep. 540(4), 167-218 (2014)

38. Kecik, K., Mitura, A., Lenci, S., Warminski, J.: Energy harvesting from a magnetic levitation system. Int. J. Non-Linear Mech. 94, 200-206 (2017)

39. Emans, J., Wiercigroch, M., Krivtsov, A.M.: Cumulative effect of structural nonlinearities: chaotic dynamics of cantilever beam system with impacts. Chaos Solitons Fractals 23(5), 1661-1670 (2005)

Publisher's Note Springer Nature remains neutral with regard to jurisdictional claims in published maps and institutional affiliations. 